\title{
Current Application of Remote Sensing Techniques in Land Use Mapping: A Case Study of Northern Parts of Kolhapur District, India
}

\author{
EHSAN GOLMEHR \\ Geosciences Department, Mississippi State University, U.S.A. \\ Flat No.12 -Fourth Floor-No.17-Shahamati Street-After Valiasr Square-Tehran-Iran,Email:ehsan.golmehr@gmail.com
}

\begin{abstract}
Land use mapping is important for evaluation, management and conservation of natural resources of an area and the knowledge on the existing land use is one of the prime pre-requisites for suggesting better use of land. In this study, we examined four mapping approaches (unsupervised, supervised, fuzzy supervised and GIS postprocessing) to identify, demarcate and map the agricultural land use categories in the Northern parts of Kolhapur district, India. A fuzzy $c$-means clustering algorithm for supervised classification approach was applied to prepare multi-layer class map and distance map. For the accuracy assessment a random stratified sampling method was used to allocate the sample size for each land use based on its spatial extent. Finally, the extracted land use map was classified into six major groups, namely forest, cultivated land, range land, waste land, water bodies and built-up land. @ JASEM
\end{abstract}

The study of land use is important not only in agriculturally dominated, over populated developing regions but throughout the world because of its relationship with different human phenomena. It has been observed that, remote sensing technique is the most efficient scientific tool in conjunction with ground truth and toposheet for collection of spatial information and very useful in identification, classification and mapping of the land use units (Nageshwara Rao and Vaidyanadhan, 1981). In case of India explosive growth of population and their diverse needs has steadily increased the need for optimum utilization of our land resources. Now the country requires sufficient quantities of food grains to feed its huge population, various raw materials for a sound industrial base and creation of adequate job opportunities for the large majority of unemployed people. The systematically planned and proper way utilization of our land resources can play a major role in solving these problems. Therefore, the proper utilization of land according to its capability should be the prime concern of people and our government. However, present research aimed to suggest the potential land use for sustainable development in the northern parts of Kolhapur district.

\section{METHODS}

Study Area

The present investigation is restricted to northern parts of Kolhapur district. The Kolhapur district is one of the 35 districts of Maharashtra and is situated in the Sahyadri hills, in the southern part of Maharashtra. The study area lies between $16^{\circ} 25^{\prime}$ and $17^{\circ} 11^{\prime}$ North latitudes and $73^{\circ} 41^{\prime}$ and $74^{\circ} 42^{\prime}$ East longitudes and is located in the Panchganga basin. East-West spread of the study region is about $107 \mathrm{~km}$ and across North-South it is about $74 \mathrm{~km}$ which covers an area of $3680.7 \mathrm{sq} \mathrm{km}$ with a population of $2,416,884$ (2006). It is included in the Pune division for administrative purpose. Total number of villages in the region is 548 . 


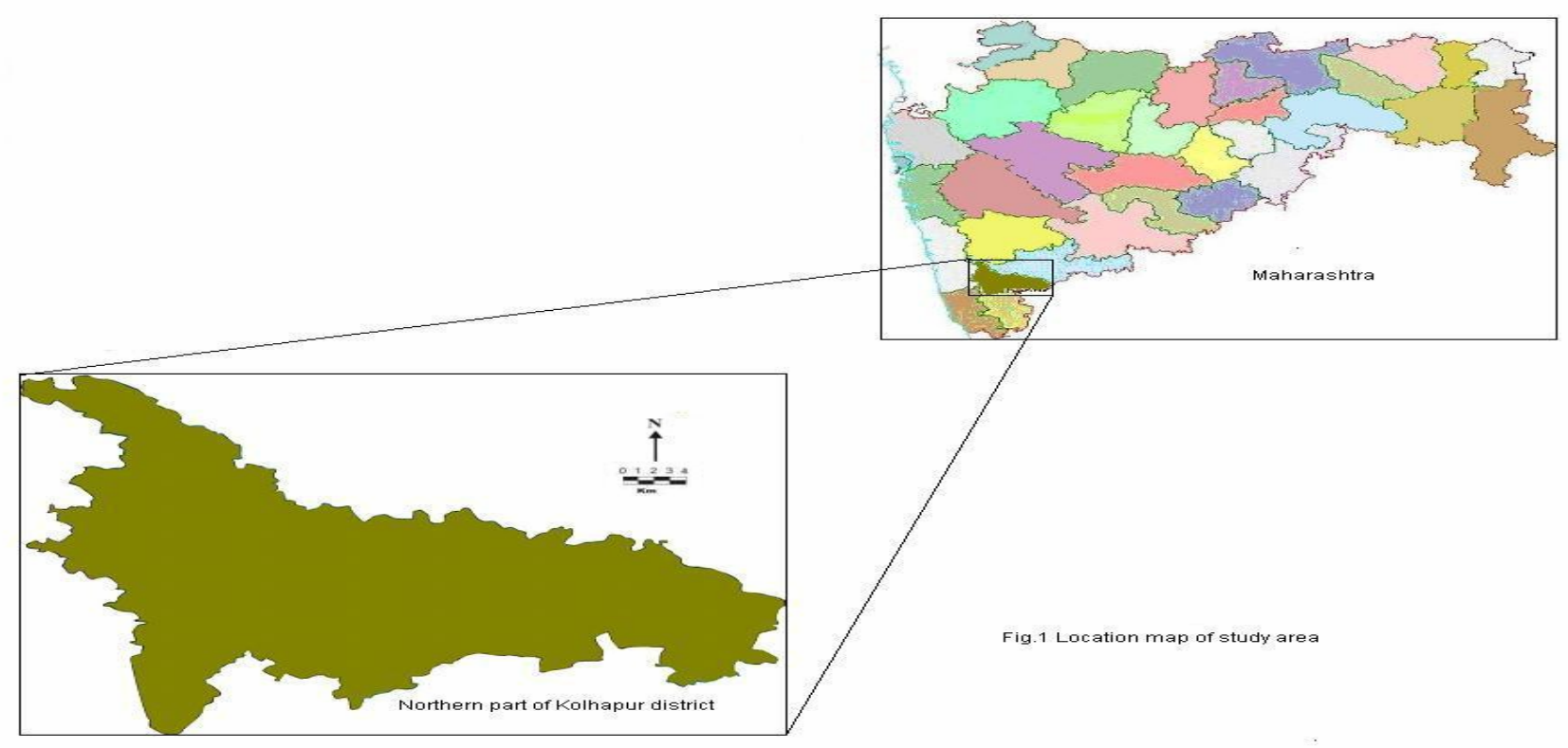

\section{Data sources}

Remote sensing image data: We used LANDSAT (spatial resolution $30 \mathrm{~m}$ ), LISS III (spatial resolution $23.5 \mathrm{~m}$ ) and ASTER data (spatial resolution $15 \mathrm{~m}$ ) for 2008 in the study. These images were selected for this study because they provided suitable cloud-free spatial coverage with relatively high spatial and spectral resolutions.

Geometric correction: Accurate registration of multispectral remote sensing data is essential for analyzing land use and land cover conditions of a particular geographic location. In this study, geometric correction of remote sensing data is done for the distortions and degradations caused by the errors due to variation in altitude, velocity of the sensor platform, variations in scan speed and in the sweep of the sensors field of view, earth curvature and relief displacement. The images were georeferenced using the polyconic projections with Root Mean Square Error (RMS) of 0.345 and 0.325 respectively. Aster and LANDSAT-7 ETM+ data were reprojected to Polyconic projections as the two data sets obtained were at Sinusoidal and UTM projections respectively.

Ground reference data: In image analysis, ground reference data play important roles to determine information classes, interpret decisions, and assess accuracies of the results (Thapa and Murayama, 2009). Substantial reference data and a thorough knowledge of the geographic area are required at this stage. In this study, we adopted both methods (primary and secondary) for collecting ground truth data. A Personal Digital Assistant (PDA) with built- in Global Positioning System (GPS) equipped with a data entry form and navigation map and a handheld digital camera were used for collecting the geographic data and recording perspective views of the locations for laboratory analysis. A total of 80 geographic locations in points and polygons and their corresponding biophysical attributes were collected in the field.

The locations of the collected data represent both the homogeneous and the heterogeneous landscape environments of the study area. In the secondary data collection method, we used higher resolution imagery acquired from airborne and spaceborne sensors, as well as city planning maps and other documents. Using all of these data, detailed ground reference data of the study area were prepared to support the land use class scheming, image classification and subsequent accuracy assessments. Furthermore, the ground reference data used for the image classification were invalid for mapping accuracy assessment purposes.

Classification scheme: Classification schemes provide frameworks for organizing and categorizing information that can be extracted from image data (Thapa and Murayama, 2007). A proper classification scheme includes classes that are both important to the study and discernible from the data on hand (Anderson, Hardy, Roach, \& Witmer, 1976). Image enhancement, contrast stretching and false colour composites were worked out and the interpretation of images were carried out using the various interpretation keys like the shape, size, pattern, tone, texture, shadows, location, association and resolution.

* Corresponding author: Ehsan Golmehr 
Using the shape man-made features as well as the natural features like the river, and hills were identified from the image. Using pattern, the airstrip, railway tracks as well as the road networks were identified. Though roads and railway tracks looks linear but both can be distinguished from each other as the major roads are associated with steep curves and many intersections with the minor roads (Joseph, 2005). Shadow shows the height of the terrain. Taller features will show larger shadows than the shorter features. Tone shows the brightness of the object based on the reflection, emittance, transmission or absorption character of an object. Using the tonal variations the vegetation status of the area was identified. Vegetated areas have a relatively high reflection in the near infrared and a low reflection in the visible range of the spectrum. Clouds, water and snow have larger visual than near infrared reflectance. Rock and bare soil have similar reflectance in both spectral regions (Cees and Farifteh, 2001).

Image Classification Techniques: The overall objective of the image classification procedure is to automatically categorize all pixels in an image into land cover classes or themes (Lillesand, Kiefer, and Chipman, 2008). Classes have to be distinguished in an image and classification needs to have different spectral characteristics. This can be analyzed by comparing spectral reflectance curves. Image classification gives results to certain level of reliability. The principle of image classification is that a pixel is assigned to a class based on its feature vector by comparing it to predefined clusters in the feature space. Doing so for all image pixels result in a classified image (Janssen. 2001). In this study unsupervised, supervised approach, fuzzy supervised and GIS post-processing were used for image classification and land use mapping of the study area.

A. Unsupervised Classification: The unsupervised classification approach is an automated classification method that creates a thematic raster layer from a remotely sensed image by letting the software identify statistical patterns in the data without using any ground truth data (Leica Geosystems, 2005; Lillesand et al., 2008). The unsupervised classification was carried out for all the three images. The spectral classes obtained from the unsupervised classification are based solely on natural groupings in the image values. The spectral classes obtained from all the three images were not initially known. So taking the reference values, the classified data was compared and the spectral classes were identified.

B. Supervised Classification: Here the image analyst supervises the pixel categorization process by

* Corresponding author: Ehsan Golmehr specifying, to the computer algorithm, numerical descriptors of various land cover types present in the image. Training samples that describes the typical spectral pattern of land cover classes are defined. Pixels in the image are compared numerically to the training samples and are labeled to land cover classes that have similar characteristics.

All the classification techniques like the maximum likelihood classification (MLC), parallelepiped and minimum distance to mean classification have been applied for the images and the best classification technique was then found out. It was observed that Maximum Likelihood Classification (MLC) was giving good results as compared to the other two techniques.

C. Fuzzy supervised classification approach: This approaches allow for multiple and partial class memberships at the level of individual pixels and accommodate fuzziness in all three stages of a supervised classification of remotely sensed imagery (Zhang and Foody 2001). This approach considers that each pixel might belong to several different classes without definite boundaries (Jensen, 2005; Wang, 1990). In this study fully-fuzzy classification which is well-known as fuzzy $c$-means clustering algorithm method is used. Where $X=\{x 1, x 2, \ldots, x \mathrm{n}\}$ a sample of $n$ observations (pixels) in an $s$-dimensional Euclidean space. Also, a fuzzy set $\left\{U_{c X n} \mid \mu_{i k} \in[0.0,1.0]\right\}$ with reference to $n$ pixels and $c$ clusters is applied. In this set $U$ is a real $c \times n$ matrix includes of $\mu_{i k}$, which is the fuzzy membership value of an observation $X_{k}$ to the ith cluster. The fuzzy membership values range from 0.0 and 1.0 and are positively related to the strength of membership of a pixel to a specified class (Zhang and Foody 2001).

In this study we used, Method of minimizing a generalized least-squared error function $i m$,

$j_{m}=\sum_{k=1}^{n} \sum_{i=1}^{c}\left(\mu_{i k}\right)^{n}\left(d_{i N}\right)^{2}$

where $m$ is the weighting exponent which controls the degree of fuzziness (increasing $m$ tends to increase fuzziness; usually, the value of $m$ is set between 1.5 and 3.0), $d^{2}$ is a measure of the distance between each observation $\left({ }^{x_{\mathrm{K}}}\right)$ and a fuzzy cluster centre $\left(v_{i}\right)$ (Bezdek et al. 1984 ). Also, the Mahalanobis distance is applied which is calculated from

$d_{i k}^{2}=\left(x_{k}-v_{i}\right)^{T} C^{-1}\left(x_{k}-v_{i}\right)$

In this algorithm $C$ is the covariance matrix of the sample $X$, and the $T$ transposition of a matrix. 
If the training pixels set $Y=\{y 1, y 2, \ldots, y \mathrm{n}\}$, and the fuzzy training data are represented as a fuzzy set $\left(G_{c X n} \mid g_{i j} \in[0.0,1.0]\right)$ with reference to $n$ training pixels and $c$ clusters, the element $g_{i j}$ represents the fuzzy membership value of a training pixel $y_{j}(1 \leq j$ $\leq n)$ to the $i$ th cluster so it is possible to define a fuzzy mean $\left({ }^{v_{t}^{*}}\right)$ and a fuzzy covariance matrix $\left({ }^{C_{t}^{*}}\right)$ (Wang, 1990). The following values were used in the classification.

$v_{\mathrm{i}}^{*}=\sum_{j=1}^{n} g_{i j} y_{j} / \sum_{j=1}^{n} g_{i j}$

$c_{i}^{*}=\sum_{j=1}^{n} g_{i d}\left(y_{j}-v_{i}^{*}\right)\left(y_{j}-v_{i}^{*}\right)^{T} / \sum_{j=1}^{n} g_{i j}$

In this way multi-layer class map and distance map were prepared.

D. GIS post-processing approach: A combination of more approaches in mapping provides better results than just using a single approach (Kuemmerle, Radeloff, Perzanowski, and Hostert, 2006; Lo and Choi, 2004). The main goal of the post-processing of raster image (after polygon generation) is an optimization of vectorization results (Lou and Huang, 2005). A GIS post-processing approach combines the three approaches (unsupervised, supervised and fuzzy supervised) to produce an improved land use and land cover map (Thapa and Murayama, 2009). The maps here are derived from the unsupervised and supervised approaches and then combined utilizing the GIS overlay function. Land use clusters identified by both unsupervised and supervised classifications produce the best results, by extracting the common land use pixels from the map. The resulting classification map revealed that the most likely homogeneous features were represented by common pixels; the more heterogeneous features were left empty. In the present study a post-classification low pass filter in the maps generated from unsupervised, supervised and GIS post-processing approaches was done before the accuracy assessment.

Accuracy assessment: In thematic mapping from remotely sensed data, the term accuracy is used typically to express the degree of 'correctness' of a map or classification (Foody, 2002). A thematic map derived with a classification may be considered accurate if it provides an unbiased representation of the land cover of the region it portrays. In essence, therefore, classification accuracy is typically taken to mean the degree to which the derived image classification agrees with reality or conforms to the 'truth' (Campbell, 1996; Janssen and van der Wel, 1994; Maling, 1989; Smits et al., 1999). A set of reference pixels representing geographic points on the classified image is required for the accuracy assessment. Randomly selected reference pixels lessen or eliminate the possibility of bias (Congalton, 1991). A random stratified sampling method was used to prepare the ground reference data. This sampling method allocates the sample size for each land use based on its spatial extent (Shalaby and Tateishi, 2007).

\section{Land Use Classification System for Northern Parts of Kolhapur District}

1.

1.1 Dense Forest

1.2 Open Forest

1.3 Forest Blank

2. Cultivated Land

2.1 Cropped Land

2.2 Plantation

2.3 Fallow Land

3. Grazing or Range Land

4. Waste Land

i. Unculturable Waste

4.1 Rocky waste

4.2 Gullied Land

ii. Culturable Waste

5. Water bodies

5.1 River

5.2 Lake/Tank

5.3 Reservoir

6. Built-up Land

6.1 Urban

6.2 Rural

6.3 Road/Railway 
Table 1: Land use (in Per cent) 2007-08

\begin{tabular}{lllllllll}
\hline Sr. No. & Tahsil & $\begin{array}{l}\text { Geographical } \\
\text { Area hectares }\end{array}$ & Forest & $\begin{array}{l}\text { Land } \\
\text { available } \\
\text { cultivation }\end{array}$ & $\begin{array}{l}\text { not } \\
\text { for }\end{array}$ & $\begin{array}{l}\text { Other } \\
\text { uncultivated } \\
\text { land }\end{array}$ & $\begin{array}{l}\text { Fallow } \\
\text { land }\end{array}$ & $\begin{array}{l}\text { Net sown } \\
\text { area }\end{array}$ \\
\hline 1 & $\begin{array}{l}\text { Gagan } \\
\text { Bavada }\end{array}$ & 28100 & 37.72 & 4.27 & 9.25 & 2.49 & 46.27 \\
2 & Shahuwadi & 104300 & 21.00 & 12.94 & & 19.27 & 7.67 & 39.12 \\
3 & Panhala & 56800 & 20.42 & 8.63 & 12.32 & 12.68 & 45.95 \\
4 & Karveer & 67100 & 1.19 & 11.92 & 13.86 & 1.79 & 71.24 \\
5 & Hatkanangal & 61000 & 2.29 & 9.51 & 5.90 & 2.30 & 80.00 \\
& e & & & & & & \\
6 & Shirol & 50800 & 1.77 & 9.45 & 5.31 & 8.47 & 75.00 \\
\hline & Total & 368100 & 14.07 & 9.45 & 10.98 & 5.9 & 59.60 \\
\hline
\end{tabular}

The land use map of northern parts of Kolhapur district adopting the above mentioned classification scheme was prepared. Map shows the distribution and extent of different land utilization units. These units could be interpreted on photographs by studying the pattern, association and variations of the image characteristics of the object in the photographs. The land use classification of northern parts of Kolhapur district has been attempted on 1:50,000 scale using FCC and black and white photographs. Many objects are inferred and identified by means of logical search and resorting to probabilities by studying and

correlating photo-elements and existing knowledge. Major portion of the study area is occupied by agricultural land use. Agricultural lands are distinguished by check-board pattern of fields, which indicates the individual field or holdings. Similarly, check-board pattern with wells indicated irrigated croplands. Built-up areas or settlement whether rural or urban are distinguished from density pattern. In this way taking into account the photo-elements, associated features and fieldwork, land use map has been prepared.

Fig.2

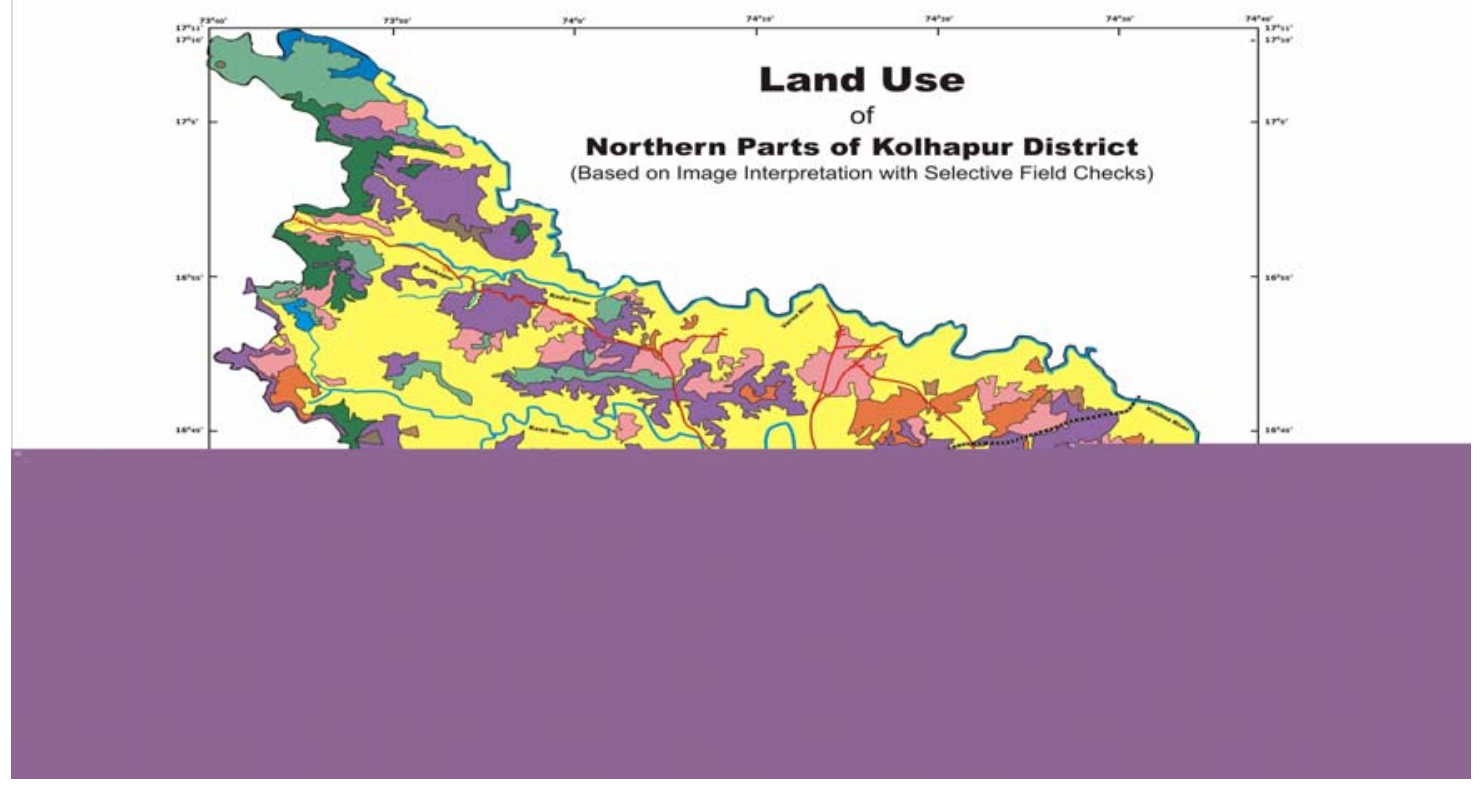

Conclusion: Assimilating spectral and radiometric properties of image data is more important than spatial resolution in improving computer-assisted land use and land cover classification accuracy. In order to improve mapping accuracies from remotely sensed data, relying on only one approach is not enough. In this study, four approaches, unsupervised, supervised, fuzzy supervised and GIS postprocessing and their accuracies to extract land use and land cover information using LANDSAT, LISS III and ASTER data for 2008 were used.

* Corresponding author: Ehsan Golmehr 
The urban woodland, water, business/industry and paddy field mapped by the GIS post-processing were observed more accurately compared to the other approaches. The fuzzy supervised approach presented slightly more accurate results than the traditional supervised approach. This method also shows great potential for dealing with heterogeneous surface features in urban residential areas showing very low difference in the errors of omission and commission. The supervised approach exhibited a lower difference between the user's and producer's accuracies for the vegetation, paddy field and water classes compared to other approaches.

The land use types of the area comprise forest, $14.07 \%$ of total surface area and about $26.33 \%$ of the area is occupied by fallow land, and uncultivated or not available land for cultivation. The remaining surface area was under cultivation.

\section{REFERENCES}

Anderson, J. R., Hardy, E. E., Roach, J. T., and Witmer, R. E., 1976, A land use and land cover classification system for use with remote sensor data. US Geological Survey Professional Paper No. 964. Washington, DC.

Campbell, J. B., 1996, Introduction to remote sensing (2nd ed.), London: Taylor and Francis.

Cees, V. W., Farifteh, J., 2001, ILWIS user's Guide. International Institute for Aerospace Survey and Earth Sciences (ITC) Enschede, Netherland, pp.43-47.

Congalton, R. G., 1991, A review of assessing the accuracy of classifications of remotely sensed data. Remote Sensing of Environment, 37, pp. 35-46.

Dutta, R., 2006, Assessment of Tea Bush Health and Yield Using Geospatial Techniques, Unpublished Thesis, IIRS, India, p.16.

Foody, M. G., 2002, Status of land cover classification accuracy assessment, Remote Sensing of Environment, 8, pp. 185- 201.

Janssen, L. L. F. a. G., 2001, Chapter 12, Digital Image Classification. Principles of Remote Sensing. International Institute for Aerospace Survey and Earth Science (ITC), Enschede, Netherlands, p.183.

Janssen, L. L. F., and van der Wel, F. J. M., 1994, Accuracy assessment of satellite derived land- cover data: a review, Photogrammetric Engineering and Remote Sensing, 60, pp.419426.

Joseph, J. 2005, Fundamentals of Remote Sensing. Hyderabad, University Press (India).

Leica Geosystems, 2005, ERDAS field guide. Norcross, Georgia: Leica Geosystems Geospatial Imaging, LLC.

Lillesand, T. M., Kiefer, R. W., and Chipman, J. W., 2008, Remote sensing and image interpretation, New York: John Wiley \& Sons, Inc.

Lou, X., and Huang, W., 2005, Raster to Vector Conversion of Classified Remote, Geoscience and Remote Sensing Symposium, IGARSS '05. Proceedings. IEEE International, pp. 36563658.

Maling, D. H., 1989, Measurements from maps. Oxford: Pergamon.

Nageshwara R., and Vaidyanadhan, R., 1981, Land Use Capability Studies from Aerial PhotoInterpretation- A Case Study from Krishna Delta, India, Geographical Review of India, 3, pp.226-236.

Shalaby, A., and Tateishi, R. ,2007, Remote sensing and GIS for mapping and monitoring land cover and land-use changes in the Northwestern coastal zone of Egypt. Applied Geography, 27, pp. $28-41$.

Smits, P. C., Dellepiane, S. G., and Schowengerdt, R. A., 1999, Quality assessment of image classification algorithms for land-cover mapping: a review and proposal for a costbased approach. International Journal of Remote Sensing, 20, pp. 1461-1486.

Thapa, R. B., \& Murayama, Y., 2007, Image classification techniques in mapping urban landscape: a case study of Tsukuba city using AVNIR-2 sensor data.Tsukuba Geoenvironmental Sciences, 3, pp. 3-10.

Thapa, R., and Murayama, Y., 2009, Urban Mapping, Accuracy, \& Image Classification: A Comparison of Multiple Approaches in Tsukuba City, Japan, Applied Geography, 29, pp.135144.

* Corresponding author: Ehsan Golmehr 